Arq. Bras. Med. Vet. Zootec., v.58, n.5, p.809-815, 2006

\title{
Características andrológicas de touros da raça Gir
}

[Andrological characteristics of Gyr (bos indicus) bulls]

\author{
I.M. Folhadella ${ }^{1,2}$, W.F. Sá ${ }^{2 *}$, A.M. Ferreira ${ }^{2}$, L.S.A. Camargo ${ }^{2}$, \\ J.H.M. Viana ${ }^{2}$, A.A. Ramos ${ }^{2,3}$, M.V.G.B. Silva ${ }^{2}$ \\ ${ }^{1}$ Universidade Federal Rural do Rio de Janeiro \\ ${ }^{2}$ Embrapa Gado de Leite \\ Rua Eugênio do Nascimento, 610 - Bairro Dom Bosco \\ 36038-330 - Juiz de Fora, MG \\ ${ }^{3}$ Universidade Federal de Minas Gerais
}

\begin{abstract}
RESUMO
Determinaram-se as características andrológicas de touros da raça Gir, classificando-os quanto ao seu potencial reprodutivo, utilizando-se o sistema de classificação andrológica por pontos (CAP). Os animais foram separados em três grupos: $\mathrm{G} 1=$ animais de $18-24$ meses $(n=33), \mathrm{G} 2=25-31$ meses $(\mathrm{n}=24)$ e G3 = 32-38 meses $(\mathrm{n}=12)$. A média da circunferência escrotal no $\mathrm{G} 1$ foi menor $(\mathrm{P}<0,05)$ do que nos $\mathrm{G} 2$ e G3. A motilidade (\%) e o vigor não diferiram entre os grupos. As taxas (\%) de defeitos maiores e totais foram maiores $(\mathrm{P}<0,05)$ no G1 do que nos G2 e G3. Para as características andrológicas não houve diferenças entre G2 e G3. Com relação ao CAP, G1, G2 e G3 apresentaram, respectivamente, 25,7\%, 58,3\% e 61,5\% de animais aptos à reprodução; $11,4 \%, 20,8 \%$ e $15,4 \%$ questionáveis e $57,1 \%, 12,5 \%$ e $15,4 \%$ inaptos ou imaturos. A idade dos touros Gir influenciou a circunferência escrotal e os defeitos maiores e totais, mas não a motilidade e o vigor. Maior proporção de animais aptos à reprodução ocorreu após 24 meses de idade.
\end{abstract}

Palavras-chave: bovino, touro, Gir, classificação andrológica

\begin{abstract}
Gyr bulls were ranked regarding their reproductive potential, following evaluations through the scorebased andrological classification system $(A C P)$. For the examinations, three animal age groups were considered: 18 to 24 month-old (G1, n=33), 25 to 31 month-old (G2, $n=24)$, and 32 to 38 month-old (G3, $n=12)$. Scrotal circumference was lower in $G 1$, as compared to $G 2$ and $G 3(P<0.05)$. Percent motility and vigor were not affected by the age groups. Percent rates of major and total defects were higher $(P<0.05)$ in $G 1$ than in $G 2$ or G3. No significant differences were observed when G2 and G3 were compared. Regarding ACP data, G1, G2 and G3 yielded, respectively, 25.7, 58.3 and $61.5 \%$ of adequate breeders; 11.4, 20.8 and 15.4\% of bulls with undefined breeding capacity, and 57.1, 12.5 and 15.4\% of inapt breeders or immature animals. Age group affected scrotal circumference and major and total defects, but did not influence motility or vigor. The highest proportion of adequate breeders was obtained with 24-month-old bulls and older.
\end{abstract}

Keywords: bovine, bull, Gyr, andrological classification system

Recebido em 12 de julho de 2004

Aceito em 7 de novembro de 2005

* Autor para correspondência (corresponding author)

E-mail: wandefsa@cnpgl.embrapa.br 


\section{INTRODUÇ̃̃O}

Pouco se sabe a respeito do comportamento reprodutivo dos touros zebuínos nas condições de campo (Kennedy et al., 2002). A maioria dos estudos que envolvem características andrológicas e classificação de touros Zebus quanto à aptidão reprodutiva baseia-se em trabalhos com a raça Nelore.

Quando se utilizam touros com valor reprodutivo e genético superior em um rebanho, pode-se reduzir o número de reprodutores em serviço e acelerar o ganho genético (Fordyce et al., 2002).

O exame andrológico é uma das técnicas mais utilizadas pelos médicos veterinários para avaliar a fertilidade. Ele caracteriza-se por exame clínico, medida de circunferência escrotal e avaliação do sêmen para motilidade, vigor, turbilhonamento, concentração e morfologia (Salvador et al., 2002). Para serem considerados reprodutivamente normais, touros zebuínos à maturidade sexual devem apresentar, no mínimo, $30 \mathrm{~cm}$ de circunferência (Chacon et al., 1999).

A motilidade, além de ser um teste simples e de rápida execução (Foote et al., 2003), está diretamente ligada à condição de manejo (Chácon et al., 2002). O teste de morfologia espermática (Saacke et al., 2000) deve ser usado como rotina, pois a disfunção física tem reflexo nas características morfológicas (Foote, 2003). Quando a incidência destes defeitos aumenta, ocorre associação com baixa fertilidade ou com imaturidade sexual (Foote, 1999).

A utilização do sistema de classificação andrológica por pontos (CAP) (Vale Filho, 1989) tem funcionado com eficiência na seleção de touros das raças zebuínas a partir de dois anos de idade (Vale Filho, 2001). Por este sistema, os touros são classificados em aptos, questionáveis e inaptos ou imaturos quanto ao seu potencial reprodutivo.

O objetivo do presente trabalho foi determinar as características andrológicas de touros jovens da raça Gir pelo exame andrológico e classificá-los quanto ao seu potencial reprodutivo, utilizandose o CAP.

\section{MATERIAL E MÉTODOS}

O trabalho foi realizado em cinco fazendas do estado de Minas Gerais, utilizando 72 touros da raça Gir, com idade variando de 18 a 38 meses. Os animais foram separados em três grupos distintos: grupo $1=$ animais de 18 a 24 meses; grupo 2= animais de 25 a 31 meses; grupo 3= animais de 32 a 38 meses. Para o exame andrológico, usou-se a técnica recomendada pelo manual de exame andrológico do Colégio Brasileiro de Reprodução Animal (CBRA) (Manual..., 1997).

Para o exame dos órgãos externos, utilizaram-se a inspeção e a palpação. Foram avaliados: escroto, testículos (circunferência escrotal), epidídimos, cordões espermáticos, prepúcio e pênis. Os órgãos internos (ampolas dos canais deferentes, glândulas vesiculares, próstata e bulbouretrais) foram examinados pela palpação retal.

O sêmen, coletado com o auxílio do eletroejaculador ${ }^{1}$, foi submetido a exames físicos e laboratoriais. Foram feitas avaliações do volume, aspecto e coloração, mediante visualização direta no recipiente e avaliações do turbilhão ou movimento de massa, motilidade, vigor, concentração e morfologia por meio de microscopia. Para avaliação do turbilhão, uma gota de sêmen, colocada sobre lâmina previamente aquecida a $37^{\circ} \mathrm{C}$, foi visualizada em microscopia ótica com aumento de cem vezes. Para motilidade e vigor, uma gota de sêmen, adicionada à lâmina previamente aquecida a $37^{\circ} \mathrm{C}$, foi diluída com citrato de sódio a $2,9 \%$ e observada em microscopia ótica com aumentos de 100 e 400 vezes. Para a concentração espermática, adicionaram-se $50 \mu \mathrm{l}$ de sêmen a $950 \mu \mathrm{l}$ de formol salina em Eppendorf, no campo. No laboratório, essa solução foi diluída de acordo com a necessidade e colocada, após a homogeneização, em câmara de Neubauer para contagem das células, usando-se a fórmula para concentração segundo o manual de exame andrológico do CBRA (Manual..., 1997).

A morfologia espermática foi estudada colocando-se uma gota de sêmen adicionada a uma gota do corante de eosina-nigrosina (Ball et al., 1983) em lâmina previamente aquecida a

${ }^{1}$ Eletrovet - Torjet ${ }^{\circledR} 65$ 
$37^{\circ} \mathrm{C}$. Após a homogeneização, fez-se um esfregaço para avaliação sob microscopia ótica, com aumento de mil vezes.

Para a classificação das patologias, foram analisados 200 espermatozóides (Manual..., 1997).

Os touros foram classificados quanto ao seu potencial reprodutivo segundo o CAP. Touros considerados aptos eram clinicamente normais e com, no mínimo, 60 pontos; touros questionáveis eram clinicamente normais e com menos de 60 pontos, e inaptos quando se apresentaram clinicamente anormais. Os imaturos foram os que apresentaram mais de $15 \%$ de defeitos maiores, $24 \%$ de defeitos menores e $30 \%$ de defeitos totais e/ou com menos de $50 \%$ de motilidade, 3 de vigor e $30 \mathrm{~cm}$ de circunferência escrotal aos 20 meses.
A análise estatística das características andrológicas foi realizada pela média dos quadrados mínimos, conforme o programa estatístico SAS (User's..., 1985), e a determinação da correlação entre as características andrológicas foi realizada pela correlação de Pearson, estimada pelo PROC CORR (User's..., 1985).

\section{RESULTADOS E DISCUSSÃO}

As médias e os erros-padrão das características andrológicas dos touros são apresentados na Tab.1. A circunferência escrotal no grupo 1 (G1) foi menor $(\mathrm{P}<0,05)$ do que nos grupos $2(\mathrm{G} 2)$ e 3 (G3). Motilidade e vigor espermáticos não diferiram entre os grupos $(\mathrm{P}>0,05)$, enquanto as taxas de defeitos maiores e totais foram maiores $(\mathrm{P}<0,05)$ no $\mathrm{G} 1$ ante $\mathrm{G} 2$ e G3.

Tabela 1. Médias dos quadrados mínimos e erros-padrão das médias das características andrológicas em touros da raça Gir de diferentes idades

\begin{tabular}{lccccc}
\hline $\begin{array}{l}\text { Idade } \\
\text { (meses) }\end{array}$ & $\begin{array}{c}\text { Circunferência } \\
\text { escrotal }\end{array}$ & Motilidade & Vigor & Defeitos maiores & Defeitos totais \\
\hline $\mathrm{G} 1$ & $28,13 \pm 0,58 \mathrm{a}$ & $58 \pm 3,9 \mathrm{a}$ & $3,2 \pm 0,2 \mathrm{a}$ & $22,1 \pm 2,5 \mathrm{a}$ & $35,3 \pm 3,2 \mathrm{a}$ \\
& $(\mathrm{n}=33)$ & $(\mathrm{n}=25)$ & $(\mathrm{n}=25)$ & $(\mathrm{n}=25)$ & $(\mathrm{n}=25)$ \\
$\mathrm{G} 2$ & $32,44 \pm 0,66 \mathrm{~b}$ & $67,6 \pm 4,1 \mathrm{a}$ & $3,5 \pm 0,2 \mathrm{a}$ & $10,9 \pm 2,6 \mathrm{~b}$ & $17,9 \pm 3,4 \mathrm{~b}$ \\
& $(\mathrm{n}=24)$ & $(\mathrm{n}=23)$ & $(\mathrm{n}=23)$ & $(\mathrm{n}=22)$ & $(\mathrm{n}=22)$ \\
$\mathrm{G} 3$ & $32,75 \pm 0,95 \mathrm{~b}$ & $69,1 \pm 6 \mathrm{a}$ & $3,6 \pm 0,3 \mathrm{a}$ & $7,5 \pm 3,8 \mathrm{~b}$ & $14,5 \pm 4,8 \mathrm{~b}$ \\
& $(\mathrm{n}=12)$ & $(\mathrm{n}=11)$ & $(\mathrm{n}=11)$ & $(\mathrm{n}=11)$ & $(\mathrm{n}=11)$ \\
\hline
\end{tabular}

G1=18 a 24 meses; G2= 25 a 31 meses; G3= 32 a 38 meses de idade.

Valores seguidos por letras distintas na mesma coluna diferiram entre si $(\mathrm{P}<0,05)$.

Os resultados para circunferência escrotal assemelham-se aos verificados para touros Brahman aos 18 meses (Vásquez et al., 2003), mas não para touros Nelore aos 24 meses (Valentim et al., 2002). Sabe-se que a circunferência escrotal depende principalmente do diâmetro e do número de túbulos seminíferos e que a variação da característica pode estar associada à idade com que os touros atingem a maturidade sexual (Valentim et al., 2002).

Cartaxo et al. (2001) observaram que animais da raça Guzerá até os 24 meses apresentaram espermatozóides com $60 \%$ de motilidade. Nas raças Canchim, Limosin e Pardo-Suíça, Oliveira et al. (2002) encontraram valores mais baixos para animais com 36 meses. Quanto ao vigor espermático, os resultados confirmam os de
Andrade et al. (2001) aos 24 meses, mas não aos 36 meses. Essas variações podem ser reflexo da estação do ano, que no presente experimento foi no final da primavera e verão, do método de avaliação, por serem análises subjetivas, da manipulação das amostras e, ainda, da proporção de patologias espermáticas existentes. Logo, a motilidade e o vigor em um simples ejaculado precisam ser interpretados cuidadosamente, levando-se em consideração também o manejo e os achados clínicos.

Os resultados da patologia espermática diferiram dos da raça Canchim aos 21 meses (Oliveira et al., 2002), e dos da raça Nelore aos 24 meses (Andrade et al., 2001), mas não dos das raças Brahman aos 18 meses (Vásquez et al., 2003) e Nelore aos 36 meses (Fonseca et al., 1997). 
A discrepância de valores encontrados na literatura e neste trabalho é, provavelmente, decorrentes das diferentes práticas de manejo impostas nos núcleos de criação. Igualmente importante, e que foi estudado neste trabalho, é a idade dos animais, não se esquecendo que na comparação entre raças, e até mesmo dentro da mesma raça, os animais atingem a maturidade sexual em diferentes épocas. Isso explica a maioria das características que diferiram do G1 para G2 e G3, mas não entre G2 e G3, uma vez que a maioria dos animais no $\mathrm{G} 1$ não atingiu a maturidade sexual. Deve-se, ainda, lembrar que os animais utilizados neste experimento foram criados no campo, originários de múltiplas propriedades, porém constituem uma amostragem representativa da média do país.

As correlações entre as características andrológicas são apresentadas na Tab. 2.

Tabela 2. Matriz de correlação e nível de significância $(\mathrm{P}<0,05)$ das características andrológicas de touros da raça Gir

\begin{tabular}{|c|c|c|c|c|c|c|c|}
\hline & Idade & $\begin{array}{l}\text { Circunf. } \\
\text { escrotal }\end{array}$ & Motilidade & Vigor & $\begin{array}{c}\text { Defeitos } \\
\text { maiores }\end{array}$ & $\begin{array}{c}\text { Defeitos } \\
\text { totais }\end{array}$ & CAP \\
\hline Idade & & $\begin{array}{c}0,64^{*} \\
0,0001^{* *} \\
(\mathrm{n}=69)\end{array}$ & $\begin{array}{c}\text { NS } \\
(n=59)\end{array}$ & $\begin{array}{c}\mathrm{NS} \\
(\mathrm{n}=59)\end{array}$ & $\begin{array}{c}-0,53 \\
0,0001 \\
(n=58)\end{array}$ & $\begin{array}{c}-0,56 \\
0,0001 \\
(n=58)\end{array}$ & $\begin{array}{c}0,4 \\
0,002 \\
(n=58)\end{array}$ \\
\hline $\begin{array}{l}\text { Circunf. } \\
\text { escrotal }\end{array}$ & & & $\begin{array}{c}0,42 \\
0,001 \\
(n=59)\end{array}$ & $\begin{array}{c}\text { NS } \\
(n=59)\end{array}$ & $\begin{array}{c}-0,49 \\
0,0001 \\
(\mathrm{n}=58)\end{array}$ & $\begin{array}{c}-0,63 \\
0,0001 \\
(\mathrm{n}=58)\end{array}$ & $\begin{array}{c}0,81 \\
0,0001 \\
(n=58)\end{array}$ \\
\hline Motilidade & & & & $\begin{array}{c}0,78 \\
0,0001 \\
(\mathrm{n}=59)\end{array}$ & $\begin{array}{c}-0,38 \\
0,004 \\
(\mathrm{n}=58) \\
\mathrm{NS} \\
(\mathrm{n}=58)\end{array}$ & $\begin{array}{c}-0,42 \\
0,0009 \\
(\mathrm{n}=58) \\
\mathrm{NS} \\
(\mathrm{n}=58)\end{array}$ & $\begin{array}{c}0,57 \\
0,0001 \\
(\mathrm{n}=58) \\
0,39 \\
0,002 \\
(\mathrm{n}=58)\end{array}$ \\
\hline $\begin{array}{l}\text { Defeitos } \\
\text { maiores }\end{array}$ & & & & & & $\begin{array}{c}0,94 \\
0,0001 \\
(\mathrm{n}=58)\end{array}$ & $\begin{array}{c}-0,73 \\
0,0001 \\
(\mathrm{n}=58)\end{array}$ \\
\hline $\begin{array}{l}\text { Defeitos } \\
\text { totais } \\
\text { CAP }\end{array}$ & & & & & & & $\begin{array}{c}-0,82 \\
0,0001 \\
(\mathrm{n}=58)\end{array}$ \\
\hline
\end{tabular}

Os resultados da correlação entre as características foram de alta, média e baixa magnitudes e foram positiva ou negativa. Para correlação alta, consideraram-se valores de 0,66 a 0,99; para média, valores de 0,33 a 0,65 e para baixa, menos de 0,33. Não se observaram correlações $(\mathrm{P}>0,05)$ entre: idade e motilidade, idade e vigor, circunferência escrotal e vigor, vigor e defeitos maiores e vigor e defeitos totais.

Os resultados da correlação entre idade e circunferência escrotal assemelham-se aos de Salvador et al. (2002), que encontraram correlação de média magnitude. Para Coe (1999) foi de baixa, porém para Brito et al. (2002), essa correlação não foi significativa. Vásquez et al. (2003) verificaram correlação de alta magnitude entre essas características.

Foi negativa e de médio o valor da correlação entre idade e defeitos maiores (Vale Filho et al., 1993).

Valentim et al. (2002) observaram baixa correlação entre circunferência escrotal e motilidade, e Martinez et al. (1999) observaram 
baixa correlação entre circunferência escrotal e vigor, diferente do que foi encontrado neste trabalho, cujas correlações foram de média magnitude e não significativas, respectivamente. Entretanto, entre circunferência escrotal e defeitos totais, os resultados deste trabalho assemelham-se aos desses autores para touros Nelore aos 20 meses de idade. Para a correlação entre motilidade e vigor, encontrou-se alta magnitude semelhante à verificada por Vale Filho et al. (1997).

O CAP foi altamente correlacionado, de forma positiva, com a circunferência escrotal, e negativa, com os defeitos maiores e totais, ou seja, quanto maior a circunferência escrotal e menores os defeitos maiores e totais, maior será o CAP. Johnson et al. (1995) encontraram correlação semelhante do CAP com a morfologia. Para a correlação entre CAP e motilidade, o resultado desta pesquisa foi de média magnitude, diferindo de Johnson et al. (1995), que encontraram alta magnitude, mas confirmando o resultado de Salvador et al. (2002), que encontraram para a mesma correlação, e para CAP e vigor, média magnitude.

As características de maior magnitude de correlação que envolveram o CAP foram: CAP e circunferência escrotal, CAP e defeitos maiores e CAP e defeitos totais. Observou-se que a avaliação dessas variáveis é de fundamental importância na determinação do potencial reprodutivo de touros Gir. A motilidade e o vigor, que também são características que controlam o CAP, não são tão importantes.

A alta correlação entre a motilidade e o vigor pode ser explicada por serem variáveis dependentes do metabolismo espermático, logo, quando ocorre alteração metabólica, ambas se modificam.

A alta correlação entre defeitos maiores e totais já era esperada, pois os defeitos totais correspondem à soma dos defeitos maiores com os defeitos menores.

A classificação de touros da raça Gir quanto à aptidão reprodutiva com base na CAP encontrase na Tab. 3.

Resultados semelhantes foram encontrados por Andrade et al. (2001) para touros Nelore de 24 meses, porém não aos 36 meses (Salvador et al., 2002). Carson e Wenzel (1997) relataram taxas de animais aptos à reprodução semelhantes às deste experimento, enquanto Higdon et al. (2000) mencionaram taxas mais altas, assim como Fonseca et al. (1997), para touros Zebu com mais de 24 meses.

Tabela 3. Classificação dos touros quanto à aptidão reprodutiva baseada no sistema de classificação andrológica por pontos (CAP)

\begin{tabular}{lcccc}
\hline $\begin{array}{l}\text { Idade } \\
\text { (meses) }\end{array}$ & Aptos & Questionáveis & Inaptos ou imaturos & $\begin{array}{c}\text { Não responderam ao } \\
\text { eletroejaculador }\end{array}$ \\
\hline Grupo 1 & $25,7 \%$ & $11,4 \%$ & $57,1 \%$ & $5,8 \%$ \\
Grupo 2 & $58,3 \%$ & $20,8 \%$ & $12,5 \%$ & $8,4 \%$ \\
Grupo 3 & $61,5 \%$ & $15,4 \%$ & $15,4 \%$ & $7,7 \%$ \\
\hline
\end{tabular}

G1 $=18$ a 24 meses; G2= 25 a 31 meses; G3= 32 a 38 meses de idade.

A classificação dos animais como questionáveis pode ser atribuída, em parte, à circunferência escrotal e/ou à motilidade/vigor enquanto as razões principais para serem classificados como imaturos foram: motilidade menor que $50 \%$, vigor menor que três, mais de $15 \%$ de defeitos espermáticos maiores, $24 \%$ de defeitos espermáticos menores, $30 \%$ de defeitos espermáticos totais e circunferência escrotal menor que $30 \mathrm{~cm}$ aos 20 meses. A classificação em inaptos ocorreu em animais com problemas clínicos.
No G1, dos 57,1\% inaptos ou imaturos, 51,4\% eram imaturos e 5,7\% inaptos devido a problemas de consistência fibrosa do epidídimo ou cisto no cordão espermático. Vale lembrar que $44,0 \%$ dos animais presentes no G1 tinham menos de 20 meses de idade. No G2, 12,5\% dos animais eram imaturos, e no $\mathrm{G} 3,7,7 \%$ eram imaturos e 7,7\% inaptos devido a problemas de edema de jarrete. 
Em geral, a taxa de reprodutores aptos quanto ao potencial reprodutivo aumentou de acordo com a idade.

\section{CONCLUSÕES}

O CAP é um sistema controlado basicamente pela circunferência escrotal e pela morfologia espermática, e bom para seleção dos touros que não apresentam distúrbios reprodutivos. A seleção andrológica de touros da raça Gir devese iniciar antes dos 18 meses, possibilitando identificar entre 18 e 24 meses animais precoces e maduros quanto ao potencial reprodutivo, para que possam ser utilizados no melhoramento genético do rebanho.

\section{REFERÊNCIAS BIBLIOGRÁFICAS}

ANDRADE, V.J.; SALVADOR, D.F.; VALE FILHO, V.R. et al. Perfil andrológico de touros da raça Nelore de dois e três anos de idade, criados extensivamente em condições do estado do Mato Grosso do Sul. Rev. Bras. Reprod. Anim., v.25, p.182-184, 2001.

BALL, L.; OTT, R.S.; MORTIMER, R.G. et al. Manual for breeding soundness of bulls. J. Soc. Theriog., v.12, p.1-65, 1983.

BRITO, L.F.C.; SILVA, A.E.D.F; RODRIGUES, L.H. et al. Effect of age and genetic group on characteristics of the scrotum, testes and testicular vascular cones, and on sperm production and semen quality in AI bulls in Brazil. Theriogenology, v.58, p.1175-1186, 2002.

CARSON, R.L.; WENZEL, J.G.W. Observations using the new bull breeding soundness evaluation forms in adult and young bulls. Vet. Clin. N. Am., v.13, p.305-311, 1997.

CARTAXO, W.O.; PENA-ALFARO, C.E.; BACALHAU, A. Parâmetros seminais e circunferência escrotal de touros jovens da raça Guzerá criados no estado da Paraíba. Rev. Bras. Reprod. Anim., v.25, p.214-215, 2001.

CHÁCON, J.; PÉREZ, E.; MÜLLER, E. et al. Breeding soundness evaluation of extensively managed bulls in Costa Rica. Theriogenology, v.52, p.221-231, 1999.
CHÁCON, J.; PÉREZ, E.; RODRÍGUEZMARTINÉZ, H. Seasonal variations in testicular consistency, scrotal circunference and spermiogramme parameters of extensively reared Brahman (Bos indicus) bulls in the tropics. Theriogenology, v.58, p.41-50, 2002.

COE, P.H. Associations among age, scrotal circunference, and proportion of morphologically normal spermatozoa in young beef bulls during an initial breeding soundness examination. $J$. Am. Vet. Med. Assoc., v.214, p.1664-1667, 1999.

FONSECA, V.O.; FRANCO, C.S.; BERGMANN, J.A.G. et al Potencial reprodutivo de touros da raça Nelore (Bos taurus indicus) acasalados com elevado número de vacas. Arq. Bras. Med. Vet. Zootec., v.49, p.53-62, 1997.

FOOTE, R. II. Fertility estimation: a review of past experience and future prospects. Anim. Reprod. Sci., v.75, p.119-139, 2003.

FOOTE, R.H. Bull sperm surface "craters" and other aspects of semen quality. Theriogenology, v. 51, p. 767-775, 1999.

FORDYCE, G.; FITZPATRICK, L.A.; COOPER, N.J. et al. Bull selection and use in Northern Australia 5. Social behaviour and management. Anim. Reprod. Sci., v.71, p.81-99, 2002.

HIGDON III, H.L.; SPITZER, J.C.; HOPKINS, F.M. et al. Outcomes of breeding soundness evaluation of 2898 yearling bulls subjected to different classification systems. Theriogenology, v.53, p.1321-1332, 2000.

JOHNSON, W.H.; THOMPSON, J.A.; KUMIDIAKA, J. et al. The determination and correlation of reproductive parameters of performance-tested Hereford and Simmental bulls. Theriogenology, v.44, p.973-982, 1995.

KENNEDY, S.P.; SPITZER, J.C.; HOPKINS, F.M. et al .Breeding soundness evaluation of 3648 yearling beef bulls using the 1993 Society for Theriogenology guidelines. Theriogenology, v.58, p.947-961, 2002.

MANUAL para exame andrológico e avaliação de sêmen animal, convênio MA/CBRA, $\mathrm{n}^{\mathbf{0}}$ 021/1997

MARTINEZ, M.L.; VERNEQUE, R.S.; TEODORO, R.L. Relações entre circunferência escrotal e a qualidade do sêmen de touros da raça Gir. REUNIÃO ANUAL DA SOCIEDADE 
BRASILEIRA DE ZOOTECNIA, 46., Anais.. Porto Alegre: SBZ, Porto Alegre. 1999.

OLIVEIRA, P.C.; BARROS, J.B.G.; CARDOSO, C.A.D. et al. Avaliação da biometria testicular e qualidade seminal em touros jovens Canchim, Limosin e Pardo Suiço. Rev. Bras. Reprod. Anim., v.26, p.61-63, 2002.

SAACKE, R.G.; DALTON, J.C.; NADIR, S. et al. Relationship of seminal traits and insemination time to fertilization rate and embryo quality. Anim. Reprod. Sci., v.60-61, p.663-677, 2000.

SALVADOR, D.F.; DIAS, J.C.; VALE FILHO, V.R. et al .Perfil andrológico de touros da raça Nelore com três e quatro anos de idade, criados extensivamente em condições do estado do Mato Grosso do Sul. Rev. Bras. Reprod. Anim., v.26, p.64-67, 2002.

USER'S guide: statistics. Version 5. Cary, NC: SAS Institute, 1985. 956p.

VALE FILHO, V.R. A evolução da andrologia veterinária no Brasil e no mundo. Cad. Tec. Vet. Zootec., n.35, p.7-14, 2001.

VALE FILHO, V.R. Padrões do sêmen bovino para o Brasil: análise e sugestões. In:
CONGRESSO BRASILEIRO DE REPRODUÇÃO ANIMAL, 8., 1989, Belo Horizonte. Anais... Belo Horizonte: CBRA, 1989. p.94-118.

VALE FILHO, V.R.; BERGMANN, J.A.G.; ANDRADE, V.J. Caracterização andrológica de touros Nelore selecionados para a primeira estação de monta. Rev. Bras. Reprod. Anim., v.21, p.42-45, 1997.

VALE FILHO, V.R.; FONSECA, V.O.; FRENEAU, G.E., Desenvolvimento testicular e maturação sexual em bovinos. Cad. Tec. Esc. Vet. UFMG, v.8, p.71-86, 1993.

VALENTIM, R.; ARRUDA, R.P.; BARNABE, R.C. et al. Biometria testicular de touros nelore (Bos taurus indicus) e touros cruzados Neloreeuropeu (Bos taurus indicus X Bos taurus taurus) aos 20 e 24 meses de idade. Braz. J. Vet. Res. Anim. Sci., v.39, p.113-120, 2002.

VÁSQUEZ, L.; VERA, O.; JÉSUS, A. Testicular growth and semen quality in peribuberal Brahman bulls. Liv. Res. Rural Develop., v.15, 2003. Disponível em: <http://www.cipav.org.co/ lrrd15/10/vasq1510.htm>. Acessado em: abril, 2006. 